\title{
Expression of Ki67 in Papillary Thyroid Microcarcinoma and its Clinical Significance
}

\author{
Yuan Zhou*, Hong-Gang Jiang, Ning Lu, Bo-Hao Lu, Zhi-Heng Chen
}

\begin{abstract}
Purpose: To investigate the expression of Ki67 protein in papillary thyroid microcarcinoma(PTMC), and to analyze its clinical significance. Materials and Methods: Ki67 protein expression was evaluated in the tissues of 108 human PTMC and 50 other benign papillary hyperplasia of thyroid specimens using immunohistochemistry. Results: The expression intensity of Ki67 in PTMC and benign papillary hyperplasia of thyroid specimens were $1.45 \pm 1.83 \%$ and $0.46 \pm 0.46 \%$.The positive expression rates were $46.3 \%$ and $14 \%$. There were significant differences between these two groups $(p<0.01)$. There was no significant variation of the expression intensity and positive expression rates of Ki67 in PTMC with gender, age, position of the tumor and the level of TSH pre-operation $(p>0.05)$, but these parameters varied with tumor size, invasion by membrane and cervical lymph node metastasis $(p<0.05$ or $p<0.01)$. Conclusions: The expression of Ki67 in PTMC was related to tumor size, invasion by membrane and cervical lymph node metastasis, and could be the important indicator for judging clinical progress and estimating prognosis.
\end{abstract}

Keywords: Papillary thyroid microcarcinoma - immunohistochemistry - Ki67

Asian Pac J Cancer Prev, 16 (4), 1605-1608

\section{Introduction}

Papillary thyroid microcarcinoma (PTMC) is one form of papillary thyroid carcinoma whose tumor size is less than 1 centimeter. In the recent years, the incidence of papillary thyroid carcinoma has upgraded significantly, especially for PTMC (Hughes et al., 2011). PTMC has very small size, is easy to be neglected and almost the prognosis is good, but we still find some PTMC cases with cervical lymph node metastasis even transfer to distant place. Cell proliferative activity is one of the important factors for assessing the biological behavior of carcinoma.

At present, Ki67 is one of the most useful markers to evaluate cell proliferative activity. It is generally used in judging clinical progress and estimating prognosis of malignant tumor nowadays. Many relevant literatures have reported that strong expression of Ki67 in many malignant tumors (Arshad et al., 2010; Choudhury et al., 2011; Chen et al., 2014). In order to investigate the expression of Ki67 in PTMC and its clinical significance, Ki67 protein expression was evaluated in the tissues of PTMC and benign papillary hyperplasia of thyroid specimens by using immunohistochemistry. We want to approach the clinical significance of Ki67 in the aspects of tumor diagnosis, clinical characteristic, prognosis of disease and treatment prescription from this study.

\section{Materials and Methods}

\section{Study objects}

A total of 158 thyroid samples from the eastern part of China collected by the department of surgical oncology and pathology in our hospital between Jan 2009 and Dec 2012 were used in this study. There were 108 cases in the PTMC group, which included 19 men and 89 women, with an average age of 47 years (range 24-76 years).

All of the PTMC cases suffered from unilateral thyroid gland lobectomy + VI lymphadenectomy. There were 30 cases with cervical lymph node metastasis, Other 78 cases were not. Tumor size was from 0.1 to 0.9 centimeter, average was 0.62 centimeter. Respectively, the benign papillary hyperplasia of thyroid specimens group contained 50 cases ( 27 cases of nodular goiter, 12 cases of thyroid adenoma, 11 cases of hashimotos thyroiditis) which included 10 men and 40 women, with an average age of 52 years (range 19-79years).

The diagnosis of thyroid disease entities was based on characteristic cytologic features and histopathological investigation.

\section{Reagents}

The antibody for Ki67, mouse monoclonal antibody sc23900, 1:100, was obtained from Santa cruz biotechnology, CA, USA.

Department of surgical oncology, Shanghai Jiao Tong University Affiliated First People's Hospital Jiaxing Branch, Jiaxing First Hospital, Jiaxing, Zhejiang, China ${ }^{\circledR}$ Equal contributors*For correspondence: yuan8422@163.com 


\section{Methods}

All specimens were fixed in $10 \%$ neutral buffered formalin ( $\mathrm{pH} 7.4$ ), dehydrated and embedded in paraffin following routine methods, cut into 4 um sections, and stained with hematoxylin and eosin (H\&E). Immunostaining has been performed by using monoclonal antibodies for Ki67. The paraffin sections were removed paraffin, and then immersed in the distilled water. Afterwards, rinsing the paraffin sections in PBS, and then blocked with $3 \%$ peroxide-methanol at room temperature for endogenous peroxidase ablation. The slides were incubated at room temperature for 2 hours with primary monoclonal antibody. After washing in PBS, the slides were incubated with biotinylated secondary antibody for 30 minutes. The reactions became visible after colouration with $\mathrm{DAB}$, kept at room temperature without light for 10 minutes. Informed consent was obtained from all patients who donated their specimens, and all the experiments were approved by the hospital ethics committee.

\section{Immunohistochemical Evaluation}

The cells were regarded as positive for Ki67 when immunoreactivity was clearly observed in their cell nucleus. Chose 10 high power visual fields randomly under microscope, count positive cell numbers in 100 tumor cells in each one filed, and than the average value was represented as the expression intensity of Ki67. According to Beesley (Beesley, 2002) classify method, immunoreactivity (no staining or weak staining) was scored as negative, immunoreactivity (staining less
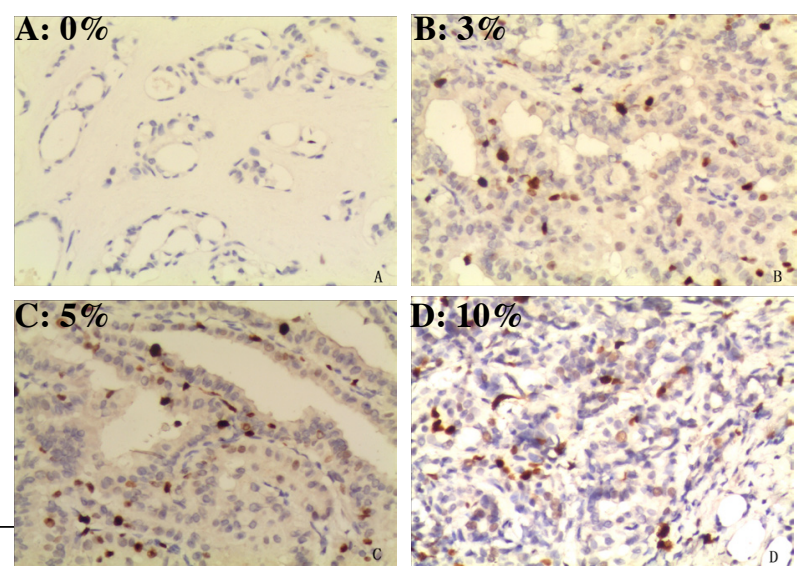

Figure 1. Expression of Ki67 in PTMC (En Vision,×200)

Table 1. Expression of Ki67 in PTMC and Benign Papillary Hyperplasia of Thyroid Specimens

\begin{tabular}{lccccccc}
\hline Type & Cases & Expression intensity $(\%)$ & $\mathrm{t}$ & $\mathrm{P}$ & Positive cases & Positive rates $(\%)$ & $\chi^{2}$ \\
\hline PTMC & 108 & $1.45 \pm 1.83$ & 5.279 & 0.000 & 50 & 46.3 & 15.458 \\
Benign & 50 & $0.46 \pm 0.46$ & & & 7 & 000 \\
\hline
\end{tabular}

Table 2. Relationship between the Clinical Characteristics and the Expression of Ki67 in PTMC

\begin{tabular}{|c|c|c|c|c|c|c|c|}
\hline \multicolumn{2}{|l|}{ Clinical characteristics } & \multirow{2}{*}{$\begin{array}{c}\text { Cases }(n) \\
19\end{array}$} & \multicolumn{2}{|c|}{ Expression intensity (\%) } & \multicolumn{2}{|l|}{$\mathrm{t}$} & $P$ \\
\hline \multirow[t]{2}{*}{ Gender } & Male & & \multicolumn{2}{|c|}{$2.40 \pm 3.10$} & \multicolumn{2}{|c|}{1.582} & 0.130 \\
\hline & Female & 89 & \multicolumn{2}{|c|}{$1.25 \pm 1.36$} & & & \\
\hline \multirow[t]{2}{*}{ Age } & $<45$ & 48 & \multicolumn{2}{|c|}{$1.75 \pm 2.32$} & \multicolumn{2}{|c|}{1.452} & 0.151 \\
\hline & $\geq 45$ & 60 & \multicolumn{2}{|c|}{$1.21 \pm 1.27$} & & & \\
\hline \multirow[t]{2}{*}{ Position of the tumor } & Upper pole & 40 & \multicolumn{2}{|c|}{$1.85 \pm 2.46$} & \multicolumn{2}{|c|}{1.519} & 0.135 \\
\hline & Not upper pole & 68 & \multicolumn{2}{|c|}{$1.21 \pm 1.28$} & & & \\
\hline \multirow[t]{2}{*}{ Level of TSH pre-operation (mU/L) } & $<4.2$ & 94 & \multicolumn{2}{|c|}{$1.30 \pm 1.41$} & \multicolumn{2}{|c|}{-1.250} & 0.232 \\
\hline & $\geq 4.2$ & 14 & \multicolumn{2}{|c|}{$2.46 \pm 3.45$} & & & \\
\hline \multirow[t]{2}{*}{ Tumor size $(\mathrm{cm})$} & $<0.5$ & 25 & \multicolumn{2}{|c|}{$0.90 \pm 1.22$} & \multicolumn{2}{|c|}{-2.198} & 0.032 \\
\hline & $\geq 0.5,<1$ & 83 & \multicolumn{2}{|c|}{$1.61 \pm 1.95$} & & & \\
\hline \multirow[t]{2}{*}{ Invasion by membrane } & Yes & 18 & \multicolumn{2}{|c|}{$2.94 \pm 3.05$} & \multicolumn{2}{|c|}{2.454} & 0.024 \\
\hline & No & 90 & \multicolumn{2}{|c|}{$1.15 \pm 1.30$} & & & \\
\hline \multirow[t]{2}{*}{ Lymph node metastasis } & Yes & 30 & $2.40 \pm$ & & \multirow{2}{*}{\multicolumn{2}{|c|}{2.631}} & 0.013 \\
\hline & No & 78 & $1.08 \pm$ & & & & \\
\hline Clinical characteristics & & Cases(n) & Positive(n) & Positive r & & $\chi^{2}$ & $P$ \\
\hline Gender & Male & 19 & 11 & 57. & & 1.247 & 0.264 \\
\hline & Female & 89 & 39 & 43. & & & \\
\hline Age & $<45$ & 48 & 21 & 43. & & 0.225 & 0.635 \\
\hline & $\geq 45$ & 60 & 29 & 48. & & & \\
\hline Position of the tumor & Upper pole & 40 & 18 & 45. & & 0.043 & 0.836 \\
\hline & Not upper pole & 68 & 32 & 47. & & & \\
\hline Level of TSH pre-operation (mU/L) & $<4.2$ & 94 & 42 & 44. & & 0.761 & 0.383 \\
\hline & $\geq 4.2$ & 14 & 8 & 57. & & & \\
\hline Tumor size (cm) & $<0.5$ & 25 & 6 & 24. & & 6.504 & 0.011 \\
\hline & $\geq 0.5,<1$ & 83 & 44 & 53. & & & \\
\hline Invasion by membrane & Yes & 18 & 14 & 77. & & 8.610 & 0.003 \\
\hline & No & 90 & 36 & 40. & & & \\
\hline Lymph node metastasis & Yes & 30 & 21 & 70 . & & 9.387 & 0.002 \\
\hline & No & 78 & 29 & 37. & & & \\
\hline
\end{tabular}


than $25 \%$ of the cells and buffy staining) was scored as weakly positive, immunoreactivity (staining 25\%-50\% of the cells and buffy staining) was scored as midrange positive, and other immunoreactivity (staining more than $50 \%$ of the cells and deep brown staining) was scored as strong positive.

\section{Follow Up}

All the cases had been followed up to observe their circumstances of recurrence, metastasis and survival. The modes of follow up included letters, telephones, outpatient clinic and so on. The deadline was Dec 30, 2013.

\section{Statistical analysis}

Statistical analysis was performed using SPSS V.17.0. Quantitative data was presented as mean $\pm \mathrm{SD}$, and the t-test was used for comparison. The $\chi^{2}$ test and Fisher exact test were used for comparison of the counting data. Differences were considered significant when $p<0.05$.

\section{Results}

Expression of Ki67 in PTMC and benign papillary hyperplasia of thyroid specimens

In this study the expression intensity of Ki67 in PTMC and benign papillary hyperplasia of thyroid specimens were $1.45 \pm 1.83 \%$ and $0.46 \pm 0.46 \%$.The positive expression rates were $46.3 \%$ and $14 \%$. There was significant difference between these two groups $(p<0.01)$ (Table 1). The expression of Ki67 in PTMC (Figure 1). Relationship between the clinical characteristics and the expression of Ki67 in PTMC

In this study we found the expression intensity and the positive expression rates of $\mathrm{Ki} 67$ in tumor size $0.5 \mathrm{~cm}$ to $1 \mathrm{~cm}$ group were higher than in tumor size less than $0.5 \mathrm{~cm}$ group and the difference between these two groups had statistical significance $(p<0.05)$. The expression intensity and the positive expression rates of Ki67 in invasion by membrane group were higher than not invasion by membrane group and the difference between these two groups had statistical significance $(p<0.05$ or $p<0.01)$. The expression intensity and the positive expression rates of Ki67 in with cervical lymph node metastasis group were higher than with no cervical lymph node metastasis group and the difference between these two groups had statistical significance $(p<0.05$ or $p<0.01)$. There were no significant difference of the expression intensity and positive expression rates of Ki67 in PTMC between gender, age, position of the tumor and the level of TSH pre-operation $(p>0.05)$. (Table 2$)$.

\section{Follow up}

The follow-up time was 11 59months, mean time was 29.7 months. There were 107 cases survived in 108 PTMC patients, including 2 cases were found cervical lymph node metastasis after surgery in 31 and 35 monthes respectively, and both of them were Ki67 positive cases. The other cases of ipsilateral recurrence, contralateral recurrence and distant metastasis were not been found. The only case of death was because of the unexpectedly cardiovascular disease.

\section{Discussion}

PTMC is a special kind of subtype of thyroid carcinoma, accounting for $10.98 \%$ 28.10\% of all the thyroid carcinoma cases (Zhang et al., 2010). Occurs in young adults, and a trend of younger, the number of female case is significantly more than men, reported in the literature, mostly in the $1: 3$ or so, some as high as $1: 9$. In this group of cases the male to female ratio is about 1:4.7. PTMC has concealed characteristics, usually considered an excellent prognosis (Jeon et al., 2009), but its biological behavior is a kind of mature cancer, part of the PTMC biological behavior is poor, can be manifested as cervical lymph node metastasis and recurrence, distant metastasis and even cause death (Zafon et al., 2010). This study displayed PTMC with cervical lymph node metastasis was $27.8 \%$.

Ki67 is a kind of DNA binding protein which presents in proliferating cell nuclear and been closely related with cell proliferation. As a marker of cell proliferation, it has been widely used in tumor treatment and research. It is mainly located in the cell nucleus, plays an important role of maintaining the structure of the DNA stable in mitosis. It has no expression in the G0 phase of the cell cycle (Haroon et al., 2013), begins to appear in the G1 phase, increases gradually in the $S$ phase and G2 phase (Tadbir et al., 2012), reaches the peak in the $\mathrm{M}$ phase, and declines rapidly in the late stage of cell division. Ki67 gene is considered as a kind of proto-oncogene at present, which locates on chromosome 10q25 and its molecular weight is 345000 (Ye et al., 2010). Ki67 has become a sensitive index of tumor cell proliferating activity. With the increasing level of the Ki67 expression, the proliferative activity of tumor cells also increase. Ki67 has the correlation with differentiation degree, tumor invasion, metastasis and prognosis of many tumors. The patients with ki67 positive expression have poor prognosis (Klintman et al., 2010). In this study, although the absolute value of expression intensity and positive rate of Ki67 in PTMC was not very high, the expression intensity was below $25 \%$, but it had statistical significance with the differences between PTMC and benign lesions with papillary hyperplasia. Some study also confirmed that Ki67 expression is beneficial for the differential diagnosis of thyroid papillary carcinoma (Aiad et al., 2013).

There is a correlation between the expression of Ki67 and the metastasis and the prognosis of many malignant tumors. This study showed that Ki67 was expressed in PTMC, its expression was related to the size of tumor, invasion by membrane and cervical lymph node metastasis, but it had nothing to do with the gender, age, position of the tumor and the level of TSH pre-operation. With the increasing of tumor size invasion by membrane and lymphatic metastasis, the expression intensity and positive rate of Ki67 were obviously increased. So it was suggested that Ki67 expression was are closely related to the tumor invasion and metastasis, Ki67 positive PTMC may have stronger ability of invasion, more prone to metastasis. In this study, the overall prognosis was good. There was no recurrence and metastasis in patients with negative expression of Ki67, but 2 cases of positive Ki67 
Yuan Zhou et al

were found cervical lymph node metastasis after surgery. It suggested that Ki67 positive patient group may be more susceptible to recurrence and metastasis after surgery. That was a group of high-risk patient population, it was needed close follow-up and in order to intervention early.

In conclusion, we have demonstrated Ki67 expressed in papillary thyroid microcarcinoma and found Ki67 was suitable biomarkers used in the distinction of PTMC from benign thyroid lesions. The expressions of $\mathrm{Ki} 67$ in PTMC was related to tumor size, invasion by membrane and cervical lymph node metastasis and could be the important indicator for judging clinical progress and estimating prognosis. Due to the limited number of patient into the group and follow-up time, it need further increasing sample and long-term follow-up to confirm the conclusion.

\section{References}

Aiad HA, Bashandy MA, Abdou AG, et al (2013). Significance of AgNORs and ki67 proliferative markers in differential diagnosis of thyroid lesions. Pthol Oncol Res, 19, 167-75.

Arshad H, Ahmad Z, Hasan SH (2010). Gliomas: correlation of histologic grade, Ki67 and p53 expression with patient survival. Asian Pac J Cancer Prev, 11, 1637-40.

Beesley MF, Mclaren KM (2002). Cytokeratin19 and galectin-3 inmunohistochemistry in the differential diagnosis of solitary thyroid nodules. Histopathology, 41, 236-43.

Chen Y, Klingen TA, Wik E, et al (2014). Breast cancer stromal elastosis is associated with mammography screening detection, low Ki67 expression and favourable prognosis in a population-based study. Diagn Pathol, 9, 230.

Choudhury M, Singh S, Agarwal S, et al (2011). Diagnostic utility of Ki67 and p53 immunostaining on solitary thyroid nodule--a cytohistological and radionuclide scintigraphic study. Indian J Pathol Microbiol, 54, 472-5.

Haroon S, Hashmi AA, Khurshid A, et al (2013). Ki67 index in breast cancer: correlation with other prognostic markers and potential in pakistani patients. Asian Pac J Cancer Prev, 14, 4353-8.

Hughes DT, Haymart MR, Miller BS, et al (2011). The most commonly occurring papillary thyroid cancer in the United States is now a microcarcinoma in a patient older than 45 years. Thyroid, 21, 231-6.

Jeon SI, Kim E, Park JS, et al (2009). Diagnostic benefit of thyroglobulin measurement in fine-needle aspiration for diagnosing metastatic cervical lymph nodes from papillary thyroid cancer correlations with US features. Korean $J$ Radiol, 10, 106-11.

Klintman M, Bendahl PO, Graban D, et a1 (2010). The prognostic value of Ki67 is dependent on estrogen receptor status and histological grade in premenopausal patients with node-negative breast cancer. Mod Pathol, 23, 251-9.

Tadbir AA, Pardis S, Ashkavandi ZJ, et al (2012). Expression of Ki67 and CD105 as proliferation and angiogenesis markers in salivary gland tumors. Asian Pac J Cancer Prev, 13, 5155-9.

Ye WJ, Xing WB, Qin LY, et al (2010). The expression and the correlational analysis of Survivin and Ki67 in the tissues of squamous cell carcinoma and basal cell epithelioma. The Chinese J Dermatovenereology, 24, 20-2.

Zafon C, Baena JA, Castellvi J, et al (2010). Differences in the form of presentation between papillary microcarcinoma and papillary carcinomas of larger size. $J$ Thyroid Res, 2011, 639156.

Zhang HX, Su C, Xie J (2010). Clinical pathology analysis of
66 cases thyroid microcarcinoma. Chinese J Cancer Prev Treat, 17, 1880-1. 